\title{
ANÁLISE DA RELAÇÃO ENTRE NÍVEIS DE COORDENAÇÃO MOTORA, APTIDÃO FÍSICA E DESEMPENHO ACADÊMIÇO EM CRIANÇAS: IMPLICAÇÕES PARA A EDUCAÇÃO FÍSICA NA ESCOLA
}

\author{
Daniel das Virgens Chagas \\ Universidade do Estado do Rio de Janeiro, Rio de Janeiro, Rio de Janeiro, Brasil \\ Dirceu Gama \\ Universidade do Estado do Rio de Janeiro, Rio de Janeiro, Rio de Janeiro, Brasil \\ Gustavo Leporace \\ Universidade Federal do Rio de Janeiro, Rio de Janeiro, Rio de Janeiro, Brasil \\ Joyce Ferreira Carvalho \\ Universidade do Estado do Rio de Janeiro, Rio de Janeiro, Rio de Janeiro, Brasil \\ Luiz Alberto Batista \\ Universidade do Estado do Rio de Janeiro, Rio de Janeiro, Rio de Janeiro, Brasil
}

\begin{abstract}
Resumo
O objetivo do estudo foi analisar a relação entre os níveis de coordenação motora, força/resistência muscular e desempenho acadêmico em crianças. A amostra foi composta por 119 escolares com idade entre 12 e 14 anos sem registros de distúrbios de aprendizagem. Os níveis de coordenação, força/resistência muscular e desempenho acadêmico foram estimados utilizando-se, respectivamente, os testes KTK, sit-up e exames governamentais. Os resultados indicaram que os níveis de força/resistência muscular podem influenciar as associações entre coordenação e desempenho acadêmico, assim como os níveis de coordenação podem influenciar as associações entre força/resistência e desempenho acadêmico. As tendências de associações positivas entre as variáveis analisadas sugerem que a prática de atividades físicas nas escolas não deve ser preterida em detrimento do alcance de metas no desempenho acadêmico.
\end{abstract}

Palavras-chave: Educação Física. Desempenho Psicomotor. Aptidão Física. Saúde na escola. Desempenho acadêmico.

\section{Introdução $^{1}$}

Há um crescente interesse público (CENTERS FOR DISEASE CONTROL AND PREVENTION, 2010) e epistêmico (TELFORD et al., 2012) na relação entre os níveis de aptidão física e o desempenho acadêmico em crianças. Nesse sentido, algumas evidências têm sustentado a existência de associações positivas entre essas variáveis (DAVIS, COOPER, 2011; SARDINHA et al., 2014; ROBERTS, FREED, MCCARTHY, 2010). Similarmente, há um crescente escopo de conhecimento indicando que adequados níveis de coordenação motora, além de serem essenciais ao desenvolvimento geral de crianças (CHAGAS,

\footnotetext{
${ }^{1} \mathrm{O}$ presente trabalho não contou com apoio financeiro de nenhuma natureza para sua realização.
} 
BATISTA, 2015; LOPES et al., 2015), também estão associados com o desempenho acadêmico (CARLSON, ROWE, CURBY; 2013; LOPES et al., 2013; SILVA et al., 2009), assim como estão associados com os níveis de aptidão física dessa população (VANDENDRIESSCHE et al., 2011).

Além das evidências que sugerem que o exercício físico estimula a plasticidade cerebral e melhora tanto a perfusão cerebrovascular quanto a função cognitiva (COTMAN, BERTCHTOLD, CHRISTIE, 2007; VOELCKER-REHAGE, NIEMANN, 2013), tem sido argumentado que crianças com maiores níveis de aptidão física têm um processamento cognitivo mais eficiente ao nível neuroelétrico, assim como maiores volumes de hipocampo e gânglios da base (HAAPALA, 2013). Ademais, as associações entre os níveis de coordenação motora e desempenho acadêmico podem ser especuladas devido ao fato de que algumas estruturas neuronais do encéfalo,como o cerebelo, o córtex frontal e os gânglios da base, são responsáveis por desempenharem funções motoras e cognitivas concomitantemente (DIAMOND, 2000; SERRIEN, IVRY, SWINNEN, 2006).

Embora haja evidências empíricas e bases teóricas sobre a existência de significativas associações entre níveis de coordenação motora e desempenho acadêmico, assim como entre níveis de aptidão física e desempenho acadêmico, são escassas as investigações analisando a relação entre essas três variáveis em crianças. Considerando as evidências sobre a existência de associações dos níveis de desempenho acadêmico com níveis de coordenação motora (CARLSON, ROWE, CURBY; 2013; LOPES et al., 2013; SILVA et al., 2009) e de aptidão física (DAVIS, COOPER, 2011; SARDINHA et al., 2014; ROBERTS, FREED, MCCARTHY, 2010), parece plausível considerar a hipótese de que os níveis de aptidão física podem influenciar as associações entre os níveis de coordenação motora e desempenho acadêmico. Do mesmo modo, os níveis de coordenação motora podem influenciar as associações entre aptidão física e desempenho acadêmico. Em nossa revisão de literatura, só foi encontrado um estudo (LOPES et al., 2013) que analisou a relação entre os níveis de coordenação motora, aptidão física e desempenho acadêmico em crianças. Entretanto, naquela oportunidade a coordenação motora foi considerada apenas como uma medida global, ou seja, não se explorou quais componentes específicos da coordenação motora estariam conduzindo as associações observadas.

Levando-se em consideração a escassez de estudos sobre o tema e a estratégia adotada em pesquisas prévias de se utilizar apenas um dos múltiplos componentes da aptidão física para estudar o fenômeno em foco nesta investigação (DAVIS, COOPER, 2011; SARDINHA et al., 2014; ROBERTS, FREED, MCCARTHY, 2010), nesta oportunidade decidiu-se analisar, especificamente, os níveis de força/resistência muscular como um dos indicadores de aptidão física. Portanto, o objetivo geral do presente estudo foi analisar a relação entre os níveis de coordenação motora, força/resistência muscular e desempenho acadêmico em crianças. Especificamente, a presente investigação se propôs a: verificar a influência dos níveis de força/resistência muscular nas associações entre os níveis de coordenação motora e desempenho acadêmico; verificar a influência dos níveis de coordenação motora nas associações entre os níveis de força/resistência muscular e desempenho acadêmico.

\section{Procedimentos Metodológicos}

Entre o sétimo e o nono ano escolar há 119 crianças (68 do sexo feminino, 51 do sexo masculino), entre 12 e 14 anos de idade, foram recrutadas para participar do presente estudo. A amostra foi extraída de uma população de estudantes de uma escola pública localizada em uma área de baixo status socioeconômico da cidade do Rio de Janeiro. Os dados demográficos dos indivíduos são fornecidos na Tabela 1 . 
Foram incluídos no estudo estudantes que ainda não tinham completado 15 anos de idade até o final da coleta de dados, sem registros de distúrbios de aprendizagem em suas fichas de cadastro escolar e/ou histórico de lesão ou doença que pudessem afetar o desempenho motor. O consentimento dos responsáveis legais, o assentimento das crianças e a aprovação pelo Comitê de Ética da Universidade (CAAE: 35534514.5.0000.5259) foram obtidos antes da participação dos indivíduos no estudo.

Todas as mensurações foram conduzidas por investigadores seguindo procedimentos padronizados, incluindo o processo de familiarização com as tarefas. As medidas foram realizadas no ginásio de esportes da escola durante o ano letivo e duraram aproximadamente 35 minutos por participante.

A massa corporal das crianças foi medida utilizando uma balança eletrônica com exatidão de $0,1 \mathrm{~kg}$ e com os participantes vestindo seus uniformes da escola. A estatura foi mensurada com as crianças descalças através de um estadiômetro de parede ao nível de $0,1 \mathrm{~cm}$.

Os níveis de coordenação motora foram mensurados utilizando o teste Körperkoordinationstest für Kinder (KTK), de Kiphard e Shilling (2007). O KTK é um instrumento válido e confiável para crianças em idade escolar e consiste de quatro tarefas: 1) equilíbrio dinâmico: caminhar para trás, equilibrando-se em barras com decrescentes larguras; 2) saltos unilaterais: ultrapassar um obstáculo formado por uma crescente barreira vertical de espumas saltando com apenas um dos membros inferiores; 3) agilidade: saltitar lateralmente sobre uma pequena trave de madeira o mais rápido possível; 4) transposição lateral: mover-se lateralmente sobre plataformas de madeira o mais rápido possível. Os escores obtidos nas quatro tarefas foram ajustados de acordo com a idade e, somados, formaram um índice global de coordenação motora.

Os níveis de força/resistência muscular foram considerados a partir dos resultados no teste sit-up em 60 segundos, oriundo da bateria de testes EuroFit (COUNCIL OF EUROPE, 1988).

Em pesquisas científicas, o desempenho acadêmico tem sido frequentemente analisado a partir de notas obtidas em testes padronizados (TOMPOROWSKI et al., 2008). Assim sendo, as notas obtidas pelas crianças em leitura, escrita e matemática em testes governamentais padronizados foram usadas para atribuir escores de desempenho acadêmico entre os participantes. Esses testes são aplicados periodicamente no sistema escolar da cidade e as notas usadas nas análises foram aquelas obtidas pelas crianças no mesmo período da coleta de dados. O grau de desempenho acadêmico poderia variar de 0 a 10, com um intervalo de um décimo (0.1).

Estatísticas descritivas foram calculadas para todas as variáveis. O teste de Kolmogorov-Smirnov confirmou aceitável normalidade de distribuição dos dados. Coeficientes de correlação de Pearson foram utilizados para examinar as associações dos níveis de desempenho acadêmico com os níveis de coordenação motora e de força/resistência muscular. Correlações parciais foram usadas para analisar as associações entre os níveis de coordenação motora e desempenho acadêmico, estatisticamente controladas pelos níveis de força/resistência muscular, assim como entre níveis de força/resistência muscular e desempenho acadêmico, controlando pelos níveis de coordenação motora. Todas as análises foram separadas por sexo. O teste de Análise de Variância (ANOVA) de um fator foi utilizado para verificar diferenças de acordo com o sexo. Um nível de significância de 5\% ( $p$ $=0.05$ ) foi adotado para todas as análises estatísticas. A análise dos dados foi realizada através do software Statistical Package for Social Sciences (SPSS ver. 22.0 software, IBM, USA). 


\section{Resultados}

Os resultados do teste de diferença e a estatística descritiva dos dados demográficos, desempenho acadêmico, coordenação motora e força/resistência muscular das crianças são apresentados na Tabela 1.

Tabela 1. Demografia dos participantes, níveis de desempenho acadêmico, coordenação motora e força/resistência muscular (média ıdesvio padrão) e níveis de diferenças entre sexos.

\begin{tabular}{|c|c|c|c|}
\hline & $\begin{array}{c}\text { Sexo } \\
\text { Masculino }\end{array}$ & $\begin{array}{c}\text { Sexo } \\
\text { Feminino } \\
\end{array}$ & p-valor \\
\hline $\begin{array}{l}\text { Idade } \\
\text { (anos) }\end{array}$ & $13.8 \pm 0.6$ & $13.8 \pm 0.7$ & \\
\hline $\begin{array}{l}\text { Índice de massa corporal } \\
\qquad\left(\mathrm{kg} / \mathrm{m}^{2}\right)\end{array}$ & $18.8 \pm 0.5$ & $21.4 \pm 0.6$ & $\mathrm{p}=0.002$ \\
\hline Coordenação global & $95.2 \pm 15.9$ & $75.9 \pm 21.2$ & $\mathrm{p}<0.001$ \\
\hline Equilíbrio Dinâmico & $101.6 \pm 15.7$ & $97.4 \pm 18.6$ & $\mathrm{p}=0.192$ \\
\hline Saltos unilaterais & $96.8 \pm 18$ & $72 \pm 27.3$ & $\mathrm{p}<0.001$ \\
\hline Agilidade & $92.7 \pm 19.3$ & $71.5 \pm 21$ & $\mathrm{p}<0.001$ \\
\hline Transposição lateral & $94.5 \pm 13.5$ & $83 \pm 17.5$ & $\mathrm{p}<0.001$ \\
\hline $\begin{array}{l}\text { Força/resistência muscular } \\
\qquad(\text { Sit- } u p)\end{array}$ & $33.4 \pm 9.3$ & $19.8 \pm 9.6$ & $\mathrm{p}<0.001$ \\
\hline $\begin{array}{c}\text { Desempenho acadêmico em } \\
\text { escrita }\end{array}$ & $6.1 \pm 2$ & $6.9 \pm 1.6$ & $\mathrm{p}=0.031$ \\
\hline $\begin{array}{c}\text { Desempenho acadêmico em } \\
\text { leitura }\end{array}$ & $6.3 \pm 2.1$ & $7.1 \pm 1.7$ & $\mathrm{p}=0.032$ \\
\hline $\begin{array}{l}\text { Desempenho acadêmico em } \\
\text { matemática }\end{array}$ & $5.9 \pm 2.2$ & $5.9 \pm 2.1$ & $\mathrm{p}=0.961$ \\
\hline
\end{tabular}

Fonte: elaboração dos autores

A coordenação global não foi significativamente correlacionada com nenhum escore de desempenho acadêmico em crianças de ambos os sexos, em ambos os tipos de análise, seja de forma separada (Tabela 2), seja controlando estatisticamente pelos níveis de força/resistência muscular (Tabela 3). Não obstante, o desempenho acadêmico em escrita se associou significativamente com os níveis de agilidade, em ambos os sexos, e com os níveis de equilíbrio dinâmico no sexo masculino (Tabela 2). Entretanto, nenhuma medida de coordenação motora foi significativamente associada com qualquer medida de desempenho acadêmico quando controlados pelos níveis de força/resistência muscular (Tabela 3).

As análises bivariadas revelaram que os níveis de força/resistência muscular e desempenho acadêmico não apresentaram associações significativas em ambos os sexos (Tabela 2). Por outro lado, as análises de correlações parciais revelaram, no sexo masculino, uma associação significativa entre os níveis de força/resistência muscular e desempenho 
acadêmico em matemática quando controlada estatisticamente por medidas de equilíbrio dinâmico (Tabela 4).

Tabela 2. Coeficientes de correlação de Pearson entre os níveis de desempenho acadêmico, coordenação motora e força/resistência muscular.

$\begin{array}{cc}\begin{array}{c}\text { Níveis de coordenação } \\ \text { motora }\end{array} & \text { Níveis de } \\ & \text { força/resistência } \\ \text { muscular }\end{array}$

\begin{tabular}{cccccccc} 
& Global & $\mathrm{ED}^{1}$ & $\mathrm{SU}^{2}$ & $\mathrm{AG}^{3}$ & $\mathrm{TL}^{4}$ & \\
\hline $\begin{array}{c}\text { Desempenho } \\
\text { acadêmico em } \\
\text { escrita }\end{array}$ & Feminino & .214 & $.256^{*}$ & .043 & $.282^{*}$ & .164 & .178 \\
$\begin{array}{c}\text { Desempenho } \\
\text { acadêmico em } \\
\text { leitura }\end{array}$ & Feminino & .068 & .108 & -.047 & .151 & 0.23 & 0.93 \\
$\begin{array}{c}\text { Desempenho } \\
\text { acadêmico em } \\
\text { matemática }\end{array}$ & Masculino & .232 & .005 & .265 & $.292^{*}$ & .079 & .158 \\
& Masculino & .109 & -.024 & .162 & .092 & .080 & .058 \\
\end{tabular}

Fonte: elaboração dos autores

${ }^{1} \mathrm{ED}=$ =quilíbrio dinâmico; ${ }^{2} \mathrm{SU}=$ saltos unilaterais; ${ }^{3} \mathrm{AG}=$ agilidade; ${ }^{4} \mathrm{TL}=$ transposição lateral.

*Correlação significativa no nível $\mathrm{p}<0.05$.

Tabela 3. Correlações parciais entre os níveis de desempenho acadêmico e coordenação motora, controlando pelos níveis de força/resistência muscular.

Níveis de coordenação motora

\begin{tabular}{ccccccc} 
& Global & $\mathrm{ED}^{1}$ & $\mathrm{SU}^{2}$ & $\mathrm{AG}^{3}$ & $\mathrm{TL}^{4}$ \\
\hline $\begin{array}{c}\text { Desempenho } \\
\text { acadêmico em } \\
\text { escrita }\end{array}$ & Feminino & .131 & .194 & -.086 & .224 & .087 \\
$\begin{array}{c}\text { Desempenho } \\
\text { acadêmico em } \\
\text { leitura }\end{array}$ & Feminino & .009 & .070 & -.134 & .224 & -.029 \\
$\begin{array}{c}\text { Desempenho } \\
\text { acadêmico em } \\
\text { matemática }\end{array}$ & Masculino & .094 & -.030 & .152 & .079 & .060 \\
\hline
\end{tabular}

Fonte: elaboração dos autores

${ }^{1} \mathrm{ED}=$ equilíbrio dinâmico; ${ }^{2} \mathrm{SU}=$ saltos unilaterais; ${ }^{3} \mathrm{AG}=$ agilidade; ${ }^{4} \mathrm{TL}=$ transposição lateral. 
Tabela 4. Correlações parciais entre os níveis de desempenho acadêmico e força/resistência muscular, controlando pelos níveis de coordenação motora.

\section{Niveis de Força/resistência muscular}

\begin{tabular}{ccc} 
& Feminino & Masculino \\
\hline & $.050^{1}$ & $.070^{1}$ \\
Desempenho & $.052^{2}$ & $.158^{2}$ \\
acadêmico em & $.192^{3}$ & $.068^{3}$ \\
escrita & $.027^{4}$ & $.078^{4}$ \\
& $.110^{5}$ & $.137^{5}$ \\
Desempenho & $.064^{1}$ & $.014^{1}$ \\
acadêmico em & $.043^{2}$ & $.061^{2}$ \\
leitura & $.155^{3}$ & $-.001^{3}$ \\
& $.012^{4}$ & $.032^{4}$ \\
Desempenho & $.095^{5}$ & $.021^{5}$ \\
acadêmico em & $-.042^{1}$ & $.246^{1}$ \\
matemática & $.031^{2}$ & $.287^{2} *$ \\
& $.020^{3}$ & $.196^{3}$ \\
& $-.045^{4}$ & $.249^{4}$ \\
\hline
\end{tabular}

Fonte: elaboração dos autores

${ }^{1} \mathrm{ED}=$ =quilíbrio dinâmico; ${ }^{2} \mathrm{SU}=$ saltos unilaterais; ${ }^{3} \mathrm{AG}=$ agilidade; ${ }^{4} \mathrm{TL}=$ transposição lateral.

*Correlação significativa no nível $\mathrm{p}<0.05$.

\section{Discussão}

O principal objetivo do presente estudo foi analisar a relação entre os níveis de coordenação motora, força/resistência muscular e desempenho acadêmico em crianças. Os resultados desta investigação sugeriram que os níveis de força/resistência muscular não influenciaram as associações entre os níveis de coordenação global e desempenho acadêmico em crianças do sexo masculino e feminino. Apesar disso, os níveis de força/resistência muscular influenciaram as associações do desempenho acadêmico em escrita com os níveis de equilíbrio dinâmico, no sexo feminino, e com os níveis de agilidade, em ambos os sexos. Ademais, os níveis de equilíbrio dinâmico influenciaram as associações entre os níveis de força/resistência muscular e desempenho acadêmico em matemática de crianças do sexo masculino.

Os resultados dos testes de correlação bivariadas e parciais sugerem que os níveis de coordenação global não estão significativamente associados com os níveis de desempenho acadêmico em crianças. Nossos achados são contrários aos de investigações prévias (CARLSON, ROWE, CURBY; 2013; LOPES et al., 2013; SILVA et al., 2009) nas quais foram sugeridas que os níveis de coordenação motora estão associados com desempenho acadêmico em crianças. Entretanto, é importante chamar a atenção para o fato de terem sido adotados diferentes desenhos de estudo nessas investigações. No estudo de Carlson, Rowe e Curby (2013), por exemplo, foram analisados níveis de coordenação motora fina, ao passo que no presente estudo foram examinados níveis de coordenação motora grossa. Na investigação de Silva et al. (2009), somente os escores de tarefas motoras classificadas pelos autores como sendo de alta demanda cognitiva apresentaram associações significativas com desempenho acadêmico em matemática de crianças. Lopes et al. (2013) estudaram as associações entre os níveis de coordenação motora grossa e desempenho acadêmico estatisticamente controladas pelos níveis de aptidão física. Entretanto, enquanto no presente 
estudo foram usadas medidas de força/resistência muscular como indicadores dos níveis de aptidão física, naquela oportunidade (LOPES et al., 2013) foi usada uma medida de condicionamento cardiovascular para controlar estatisticamente as associações entre os níveis de coordenação motora e desempenho acadêmico em crianças. De fato, é difícil comparar nossos achados com aqueles oriundos de outros estudos devido não só aos diferentes tipos de desenhos adotados entre as pesquisas, mas também à escassez de investigações sobre o tema. Logo, estudos adicionais devem ser conduzidos para verificar se associações entre os níveis de coordenação global e desempenho acadêmico não apresentam, de fato, significância estatística quando controladas por níveis de força/resistência muscular em crianças.

Não obstante, nossos achados sugerem que as associações entre os níveis de coordenação motora e desempenho acadêmico podem variar de acordo com as medidas utilizadas como indicadores de coordenação, visto que o desempenho acadêmico em escrita se correlacionou positivamente com os níveis de equilíbrio dinâmico, em meninas, e agilidade, em ambos os sexos. Entretanto, os resultados das correlações parciais revelaram que tais associações não alcançaram significância estatística quando controladas pelos níveis de força/resistência muscular. Logo, esses achados sugerem que os níveis de força/resistência muscular poderiam influenciar as associações entre os níveis de coordenação motora e desempenho acadêmico em crianças.

Outro aspecto investigado neste estudo foi a associação entre os níveis de força/resistência muscular e desempenho acadêmico em crianças. De um modo geral, a literatura sugere que os níveis de condicionamento cardiovascular e desempenho acadêmico estão positivamente associados em escolares (BASS et al., 2013; DAVIS, COOPER, 2011; HAAPALA, 2013; ROBERTS, FREED, MCCARTHY, 2010; SARDINHA et al., 2014.), embora alguns estudos não ratifiquem plenamente essa assertiva (EVELAND-SAYERS et al., 2009; KWAK et al., 2009; TELFORD et al., 2012). Para Torrijos-Niño et al. (2014), as associações positivas entre níveis de aptidão física e desempenho acadêmico estão restritas aos níveis de condicionamento cardiovascular, e não aos níveis de força muscular. Outras evidências (ESTEBAN-CORNEJO et al., 2014; AGUILAR et al., 2015) corroboram tal suposição. Especificamente no que diz respeito à análise dos níveis de força/resistência muscular, os resultados das análises bivariadas do presente estudo estão alinhados com essas evidências (ESTEBAN-CORNEJO et al., 2014; TORRIJOS-NIÑO et al., 2014; AGUILAR et al., 2015), uma vez que não foram encontradas associações significativas entre níveis de força/resistência muscular e desempenho acadêmico em escolares.

Contrário aos nossos achados, estudos prévios sugerem uma associação positiva entre os níveis de força/resistência muscular e desempenho acadêmico em leitura, escrita e matemática em crianças (BASS et al., 2013; CHOMITZ et al., 2009; COE et al., 2013). Em contrapartida, em outros estudos (EVELAND-SAYERS et al., 2009; VAN DER NIET et al., 2014) foram encontradas associações positivas entre níveis de força/resistência muscular e desempenho acadêmico em matemática, mas não em leitura e escrita, em escolares do Ensino Fundamental. Portanto, a divergência de resultados entre os estudos sugere que ainda não está bem estabelecida a existência de associações entre os níveis de força/resistência muscular e desempenho acadêmico em crianças. Para além da divergência dos resultados, novamente é importante destacar que nos estudos acima discutidos foram adotados distintos procedimentos de investigação.

Um dos aspectos metodológicos que pode ter enviesado resultados prévios sobre a associação entre níveis de força/resistência muscular e desempenho acadêmico está relacionado ao controle estatístico de covariáveis. Nesse sentido, os achados do presente estudo sugerem que os níveis de equilíbrio dinâmico influenciaram a associação entre os níveis de força/resistência muscular e desempenho acadêmico em matemática de crianças do 
sexo masculino, uma vez que tal associação passou a apresentar significância estatística quando controlada pela covariável equilíbrio dinâmico.

Os achados do presente estudo sugerem que a relação entre os níveis de coordenação motora, aptidão física e desempenho acadêmico em crianças é complexo e pode variar de acordo com as variáveis utilizadas nas análises associativas. Enquanto as associações entre os níveis de coordenação motora e desempenho acadêmico deixaram de apresentar significância estatística quando controladas por níveis de força/resistência muscular, o oposto foi observado nas associações entre os níveis de força/resistência muscular e desempenho acadêmico quando controlados pelos níveis de equilíbrio dinâmico. Investigações futuras deveriam considerar outras variáveis de controle, tais quais os níveis de condicionamento cardiovascular, no estudo das associações envolvendo coordenação motora, aptidão física e desempenho acadêmico.

Por fim, é importante considerar o atual contexto socioeducacional de países em desenvolvimento, como o Brasil, que têm estabelecido metas em desempenho acadêmico para suas escolas públicas como uma medida para aprimorar seus índices de desenvolvimento humano. Com a crescente preocupação em aumentar os níveis de desempenho acadêmico, o tempo dedicado às atividades físicas, que são fundamentais para o desenvolvimento da coordenação motora e da aptidão física em crianças, diminuiu significativamente nas últimas décadas em escolas públicas (DONNELLY; LAMBOURNE, 2011). Entretanto, além do desestímulo ao desenvolvimento da coordenação e da aptidão física ter um impacto negativo na saúde dos escolares, é importante destacar que a relação entre os níveis de coordenação motora, força/resistência muscular e desempenho acadêmico em crianças mostraram tendências de associações positivas. Cabe ressaltar, ainda, uma recente investigação realizada por Peserico et al. (2015), cujas evidências sugerem que o tempo dedicado à prática esportiva extraclasse não prejudicou o aproveitamento escolar de estudantes entre 13 e 17 anos.

No presente estudo foi constatado que os níveis de força/resistência muscular podem influenciar as associações entre os níveis de coordenação motora e desempenho acadêmico em crianças. Similarmente, foi observado que os níveis de coordenação motora, especificamente o equilíbrio dinâmico, podem influenciar as associações entre os níveis de força/resistência muscular e desempenho acadêmico. Para além das questões relacionadas à saúde, as tendências de associações positivas entre os níveis de coordenação motora, força/resistência muscular e desempenho acadêmico em crianças sugerem que os gestores educacionais não devem preterir a prática de atividades físicas nas escolas em detrimento do alcance de metas no desempenho acadêmico.

\title{
ANALYSIS OF THE RELATIONSHIP AMONG LEVELS OF MOTOR COORDINATION, PHYSICAL FITNESS, AND ACADEMIC ACHIEVEMENT IN CHILDREN: IMPLICATIONS FOR PHYSICAL EDUCATION IN THE SCHOOL
}

\begin{abstract}
This study aimed to analyze the relationships among motor coordination, physical fitness, and academic achievement among children. The sample consisted of 119 students aged between 12 and 14 years old without medical certificate of learning disabilities. Motor coordination, physical fitness, and academic achievement were estimated through KTK, sit-up, and governmental tests respectively. Results suggest that muscular strength/endurance may influence the associations between coordination and academic achievement, as well as the coordination levels may influence the associations between muscular strength/endurance and academic achievement. The trends of positive associations among these variables suggest that
\end{abstract}


engagement in physical activity should not be discouraged due to goals in academic achievement.

Keywords: Physical Education. Psychomotor Performance. Physical Fitness. Health at School. Academic Achievement.

\section{ANÁLISIS DE LA RELACIÓN ENTRE LOS NIVELES DECOORDINACIÓN MOTORA, HABILIDAD FÍSICA Y RENDIMIENTO ACADÉMICO EN NIÑOS: IMPLICACIONES PARA LA EDUCACIÓN FÍSICA EN LA ESCUELA}

\section{Resumen}

El objetivo de estudio fue analizar la relación entre los niveles de coordinación motora, fuerza/resistencia muscular y rendimiento académico en niños. La muestra se estuvo compuesta por 119 alumnos con edades comprendidas entre 12 y 14 años y sin registros de trastornos de aprendizaje. Los niveles de coordinación, fuerza/resistencia muscular y rendimiento académico fueron estimados usándose, respectivamente, KTK, sit-up, y las pruebas gubernamentales. Los resultados sugieren que los resultados de fuerza/resistencia muscular pueden influenciar las asociaciones entre coordinación y rendimiento académico, así como los niveles de la coordinación y desempeño pueden influenciar las asociaciones entre fuerza/resistencia y rendimiento académico. Las tendencias de las asociaciones positivas entre las variables sugieren que la práctica de actividades físicas en las escuelas no debe ser restringida en detrimiento del logro de los objetivos en el rendimiento académico.

Palabras-clave: Educación Física. Desempeño Psicomotor. Habilidad Física. Salud Escolar. Rendimiento Académico.

\section{Referências}

AGUILAR, M.M.; VERGARA, F.A.; VELÁSQUEZ, E.J.A.; MARINA, R.; GARCÍAHERMOSO,A. Screen time impairs the relationship between physical activity and academic attainment in children. Jornal de Pediatria, Rio de Janeiro, v. 91, n.4, p. 339-345, 2015.

BASS, R.W.; BROWN, D.D.; LAURSON, K.R.; COLEMAN, M.M. Physical fitness and academic performance in middle school students. Acta Paediatrica, v. 102, n. 8, p. 832-837, 2013.

CARLSON, A.G.; ROWE, E.; CURBY, T.W. Disentangling fine motor skills' relations to academic achievement: the relative contributions of visual-spatial integrations and visualmotor coordination. The Journal of Genetic Psychology, v. 174, n. 5-6, p. 514-533, 2013.

CENTERS FOR DISEASE CONTROL AND PREVENTION. The association between school-based physical activity, including physical education, and academic performance. Atlanta, GA: U.S. Department of Health and Human Services, 2010.

CHAGAS, D.V.; BATISTA, L.A. Interrelationships among motor coordination, body fat percentage, and physical activity in adolescent girls. Human Movement, v. 16, n. 1, p. 4-8, 2015.

CHOMITZ, V.R.; SLINING, M.M.; MCGOWAN, R.J.; MITCHELL, S.E.; DAWSON, G.F.; HACKER, K.A. Is there a relationship between physical fitness and academic 
achievement? Positive results from public school children in the northeastern United States. Journal of School Health, v. 79, p. 30-37, 2009.

COE, D,P.; PETERSON, T.; BLAIR, C.; SCHUTTEN, M.C.; PEDDIE,H. Physical fitness, academic achievement, and socioeconomic status in school-aged youth. Journal of School Health, v. 83, p. 500-507, 2013.

COTMAN, C.; BERTCHTOLD, N.; CHRISTIE,L. A. Exercise builds brain health: key roles of growth factor cascades and inflammation. Trends in Neuroscience, v.30, p. 464-472, 2007.

COUNCIL OF EUROPE: EUROFIT. Handbook for the Eurofit Tests of Physical Fitness. Rome: Council of Europe, 1988.

DAVIS, C.; COOPER,S. Fitness, fatness, cognition, behavior, and academic achievement among overweight children: Do cross-sectional associations correspond to exercise trial outcomes? Preventive Medicine, v.52, suplemento 1, p. S65-S69, 2011.

DIAMOND, A. Close interrelation of motor development and cognitive development and of the cerebellum and prefrontal cortex. Child Development, v.71, p. 44-56, 2000.

DONNELLY, J.E.; LAMBOURNE, K. Class-room based physical activity, cognition, and academic achievement. Preventive Medicine, v.52, p. S36-S42, 2011.

ESTEBAN-CORNEJO, I.; TEJERO-GONZÁKES, C.M.; MARTINEZ-GOMEZ, D.; DELCAMPO, J.; GONZÁLEZ-GALO, A.; PADILLA-MOLEDO, C. et al. Independent and combined influence of the components of physical fitness on academic performance in youth. Journal of Pediatrics, v.165, p. 306-312, 2014.

EVELAND-SAYERS, B.; FARLEY, R.; FULLER, D.; MORGAN, D.; CAPUTO,J. Physical fitness and academic achievement in elementary school children. Journal of Physical Activity and Health, v.6, p. 99-104, 2009.

HAAPALA, E.Cardiorespiratory fitness and motor skills in relation to cognition and academic performance in children: a review. Journal of Human Kinetics, v.36, p. 55-68, 2013.

HILLMAN, C.; PONTIFEX, L.; RAINE, L.; CASTELLI, D.; HALL, E.; KRAMER, A. The effect of acute treadmill walking on cognitive control and academic achievement in preadolescent children. Neuroscience. v.159, p. 1044-1054, 2009.

KIPARD, E.; SHILLING,F. Körperkoordinationstest für Kinder. Göttingen: Hogrefe, 2007.

Kwak L, Kremers A, Bergman P, Ruiz J, Rizzo N, Sjöström M. Associations between physical activity, fitness, and academic achievement. Journal of Pediatrics, v.155, p. 914918, 2009. 
LOPES, L.; SANTOS, R.; MOREIRA, C.; PEREIRA, B.; LOPES, V. Sensitivity and specificity of different measures of adiposity to distinguish between low/high motor coordination. Jornal de Pediatria, Rio de Janeiro, v. 91, p. 44-51, 2015.

LOPES, L.; SANTOS, R.; PEREIRA, B.; LOPES,V. Associations between motor coordination and academic achievement in elementary school children. Human Movement Science,v. 32, p. 9-20, 2013.

PESERICO, C.S.; KRAVCHYCHYN, C.; OLIVEIRA, A.A.B. Análise da relação entre esporte e desempenho escolar: um estudo de caso. Pensar a Prática, Goiânia, v. 18, n.2, p. 1$16,2015$.

ROBERTS, C.; FREED, B.; MCCARTHY,W. Low aerobic fitness and obesity are associated with lower standardized test scores in children. Journal of Pediatrics, v. 156, p. 711-718, 2010.

SARDINHA, L.; MARQUES, A.; MARTINS, S.; PALMEIRA, A.; MINDERICO, C. Fitness, fatness, and academic performance in seventh-grade elementary school students. BMC Pediatrics. v. 14, p. 176-184, 2014.

SERRIEN, D.J.; IVRY, R.B.; SWINNEN, S.P. Dynamics of hemispheric specialization and integration in the context of motor control. Nature ReviewsNeuroscience, v. 7, p. 160-166, 2006.

SILVA, C.M.M.; CARMO, A.J.; MORALES, A.P.; SILVA, V.F. Multidisciplinaridade na prática: a relação entre educação física e matemática e suas colaborações mútuas no desenvolvimento cognitivo de escolares da educação infantil. Pensar a prática, Goiânia, v.12, n. 2, p. 1-14, 2009.

TELFORD, R.D.; CUNNINGHAM, R.B.; FITZGERALD, R.; OLIVE, L.S.; PROSSER, L.; JIANG, X. et al. Physical education, obesity, and academic achievement: a 2-year longitudinal investigation of Australian elementary school children. American Journal of Public Health, v. 102, p. 368-374, 2012.

TELFORD, R.D.; CUNNINGHAM, R.B.; TELFORD, R.M.; ABHARATNA, W.P. Schools with fitter children achieve better literacy and numeracy results: evidence of a school cultural effect. Pediatric Exercise Science, v. 24, p. 45-57, 2012.

TOMPOROWSKI, P,; DAVIS, C.; MILLER, P.; NAGLIERI,J. Exercise and children's intelligence, cognition, and academic achievement. Educational Psychological Review, v. 20, p. 111-131, 2008.

TORRIJOS-NIÑO, C.; MARTÍNEZ-VIZCAÍNO, V.; PARDO-GUIJARRO, M.J.; GARCÍAPRIETO, J.C.; ARIAS-PALENCIA, N.M.; SÁNCHEZ-LÓPEZ, M.Physical fitness, obesity, and academic achievement in schoolchildren. Journal of Pediatrics, v.165, n.1, p. 104-9, 2014.

VANDENDRIESSCHE, J.; VANDORPE, B.; COELHO-E-SILVA, M.; VAEYENS, R.; LENOIR, M.; LEFEVRE,J. et al. Multivariate association among morphology, fitness, and 
motor coordination characteristics in boys age 7 to 11. Pediatric Exercise Science, v. 23, p. 504-520, 2011.

VAN DER NIET, A.G.; HARTMAN, E.; SMITH, J.; VISSCHER,C. Modeling relationships between physical fitness, executive functioning, and academic achievement in primary school children. Psychology of Sport and Exercise ,v. 15, p. 319-325, 2014.

VOELCKER-REHAGE, C.; NIEMANN, C. Structutal and functional brain changes related to different types of physical activity across the life span. Neuroscience \& Biobehavioral Review. v. 37, p. 2068-2295, 2013.

Endereço para correspondência:

chagas_daniel@yahoo.com.br

Daniel das Virgens Chagas

Universidade do Estado do Rio de Janeiro, Instituto de Educação Física e Desportos. Av. São Francisco Xavier, 524. Ginásio de Esportes, Laboratório de Biomecânica e Comportamento Motor.

Maracanã

20550-900 - Rio de Janeiro, RJ - Brasil 